\title{
Interaction Between Colloidal Quantum Dots and Halide Perovskites: Looking for Constructive Synergies
}

\author{
Thi Tuyen Ngo, ${ }^{1}$ and Iván Mora-Seró ${ }^{1, *}$
}

${ }^{1}$ Institute of Advanced Materials (INAM), Universitat Jaume I, 12006 Castelló, Spain.

*Corresponding Authors: sero@uji.es

\begin{abstract}
Colloidal Quantum Dots (QDs) have received an extensive attention during the last few decades due to their amazing properties emerging from the quantum confinement. In parallel, halide perovskites have attracted attention by the demonstration of very high performances especially in solar cell and LEDs, and other optoelectronic devices. Both families of materials can be prepared in a relatively simple way, facilitating their integration. There are several examples of their interaction enhancing the properties of the final nanocomposite. Perovskite can effectively passivate QDs, or act as efficient charge transporters. While QDs can be used to modify the selective contacts in perovskite devices or can be used as efficient light emitters or absorbers for enhanced LEDs and Photodetectors, respectively. Moreover, QD can seed the perovskite crystal growth, improving the morphology and ultimately the solar cell performance. In addition, new advanced devices can appear by the constructive synergy between both families of materials.
\end{abstract}

\section{TOC}

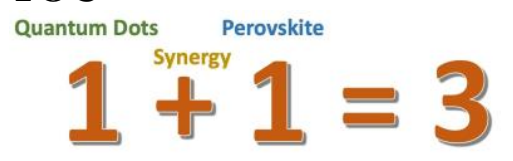

\section{Quotes}

- Combinations of materials with different nature has been a successful strategy in order to develop new materials with enhanced properties.

- The combination of halide perovskites and colloidal quantum is a promising strategy looking for constructive synergies where the whole is more than the sum of the parts.

- Combination of halide perovskites and colloidal quantum have improved the performance of solar cells, LEDs and photodetectors.

- Advanced configurations as tunable LEDs and Intermediate Band Gap Solar cells could be potentially implemented if non-radiative recombination can be avoided. 
Combinations of materials with different nature has been a successful strategy in order to develop new materials with enhanced properties. Adobe, concrete, stained glass, stainless steel are just some examples of how this strategy has provided some of the most employed materials that has been used for the humankind for centuries even for millennia. This strategy is still useful nowadays when the development of chemistry, quantum mechanics and nanotechnology has created a revolution of material science. Two paradigmatic relatively new materials with a clear applicability in optoelectronics are halide perovskites (HPs) and semiconductor quantum dots (QDs). Both families of materials are exciting by themselves and they are somewhat easy to combine, consequently there is a huge interest in building up constructive synergies in order to obtain properties and/or devices with enhanced functionalities and performance by their combined use.

Halide Perovskite (HP) is a family of materials with outstanding versatility not only for the preparation of high efficiency solar cells, with certified efficiencies higher than $23 \%,{ }^{1}$ but also photodetectors, ${ }^{2}$ light emitting diodes (LEDs), ${ }^{3-6}$ light amplifier ${ }^{7-8}$ and lasers. ${ }^{9-12}$ Although HPs has been studied in the past, ${ }^{13}$ the interest for these materials has reached its maximum level in the last few years. This interest has been mainly originated by the impressive photovoltaic conversion efficiencies reported in perovskite solar cells (PSCs) and how this efficiency has increased quickly from $10 \%$ to $23 \%$ in less than six years. ${ }^{1}{ }^{14-18}$ In PSCs it has been shown that HP not only acts as excellent light harvester ${ }^{19}$ but also as Electron Transporting Material (ETM), ${ }^{14}$ Hole Transporting (HTM),${ }^{20}$ or both in planar devices with no mesoporous scaffold. ${ }^{21}$ Perovskite is able to accumulate charge by itself, ${ }^{22}$ constituting a new class of photovoltaic device differentiated from its ancestor, the dye sensitized solar cells. Additionally HPs can be prepared from solution methods at low temperatures and, consequently, using low cost fabrication techniques. Solution processes also allows HPs to combine easily with other materials such as organic compounds ${ }^{23}$ and QDs. ${ }^{23-26}$

On the other hand, colloidal QDs offer a high versatility with tunable bandgap just by size or shape control, due to quantum confinement effect, pure color and bright emission, and generally better stability than organic chromophores. ${ }^{5,27-28}$ Consequently, QDs have attracted considerable attention due to their unique and suitable electro-optical properties for the application of QDs in LEDs, ${ }^{27,29-31}$ solar cells ${ }^{32-38}$ and other optoelectronic devices. ${ }^{27}$ Besides, solar cells prepared with QD thin films have demonstrated certified photovoltaic conversion efficiencies as high as $13.4 \%,{ }^{39}$ while for sensitized configuration efficiencies close to $13 \%$ have been reached. ${ }^{40}$

Considering all these outstanding properties for HPs and colloidal QDs the combination of both systems is a promising strategy looking for constructive synergies where the whole is more than the sum of the parts. There are previous examples of constructive combination of different family materials as molecular dyes and colloidal QDs that can provide enhanced charge photoinjection into $\mathrm{TiO}_{2}{ }^{41}$ and consequently increase the performance of the sensitized solar cells. ${ }^{42}$ In this case, the obtained photocurrent is not just the consequence of the addition of 
materials absorbing at different light wavelengths but higher. The internal recombination inside the QDs of the photogenerated carriers before being injected is highly suppressed due to a fast hole extraction towards the attached dye. ${ }^{42}$ This is a clear example of a constructive synergy.

As both HPs and QDs are promising for optoelectronic devices, their combination could benefit of the advantages of these two materials such as the strong luminescent efficiency of QDs and long range charge carrier transport of HPs. Here we summarize important studies on the interaction between HPs and QDs which has been applied for the development of optoelectronic devices such as solar cells ${ }^{43-46}$, LEDs ${ }^{25-26,47-48}$ and photodetectors, ${ }^{49-51}$ and discuss the perspectives of the synergistic interaction between them.

HPs and QDs can be fabricated using relatively simple synthetic routes allowing an easy integration of both materials and consequently favored the apparition of different works which explored the integration of both materials after the breakthrough of the first reports on all-solid PSCs. Different QDs have been investigated in order to combine with HPs. The PbS QDs have been likely the most employed ones due to their bandgap that can be tuned deeper in the IR than HPs and also due to the relatively low lattice mismatch. ${ }^{24}$ In fact the lattice mismatch can be further reduced by the use of mixed halide I-Br perovskite., ${ }^{48}$ or the use of inorganic perovskites. ${ }^{52}$ Researchers have combined HPs with colloidal QDs following different objectives as the use of the selective and transport properties of one of the compounds, attain a broader light absorption range, the passivation of QD surface or the implementation of advanced solar cell or LED configurations.

QDs have been used as HTM in PSCs. Having a proper band energy alignment is a key parameter to achieve a good performance of optoelectronic devices in general and solar cells in particular. $\mathrm{Hu}$ et al. synthesized PbS QDs with tuned bandgap from 0.7 to $2.1 \mathrm{eV}$ and used them as HTM in PSCs with a structure of ITO/PbS QDs/MAPbI $3 / \mathrm{PCBM}_{3} / \mathrm{Al}^{43}{ }^{43} \mathrm{By}$ tuning the size of $\mathrm{PbS}$ colloidal QDs, they engineered the energy alignment between PbS QD acting as HTM and HP active layer. The use of PbS QDs HTM allowed to harvest photons up to $1000 \mathrm{~nm}$ wavelength and increased significantly the performance respect cells with no HTM but far of the efficiency obtained with the most common HTMs. ${ }^{43}$

Co-sensitization of HPs and QDs is one of the possibilities that have awaken more interest regarding the combination of the two material families. In this combination the purpose is to use the broader absorption range of the PbS QDs in the IR with the good transport properties of HPs. The combination of HPs and QDs has been used as a co-sensitizer in a heterojunction solar cells. $\mathrm{CH}_{3} \mathrm{NH}_{3} \mathrm{PbI}_{3}$ $\left(\mathrm{MAPbI}_{3}\right)$ has an excellent absorption of the solar spectrum in the visible range but not in the near infrared range because of its bandgap $(1.57 \mathrm{eV})$. The use of cosensitizer HP and QDs can overcome this absorption limitation as suggested the first time by Etgar et al. ${ }^{44}$ QDs act as an absorber in NIR region, see Figure 1a, resulting a higher short circuit current density, $J_{s c}$, than for single $\mathrm{MAPbI}_{3}$ devices, ${ }^{44,53-54}$ with contribution to the photocurrent of photons harvested by the two co-absorbers as it is observed from IPCE measurements, see Figure 1b. On the 
other hand, the study of Vinh et al. pointed out a synergistic effect of the addition of $\mathrm{PbS} \mathrm{QDs}$ as it induced hydrophobic modification of the perovskite surface, leading to an improvement of the device stability in air atmosphere. ${ }^{54}$
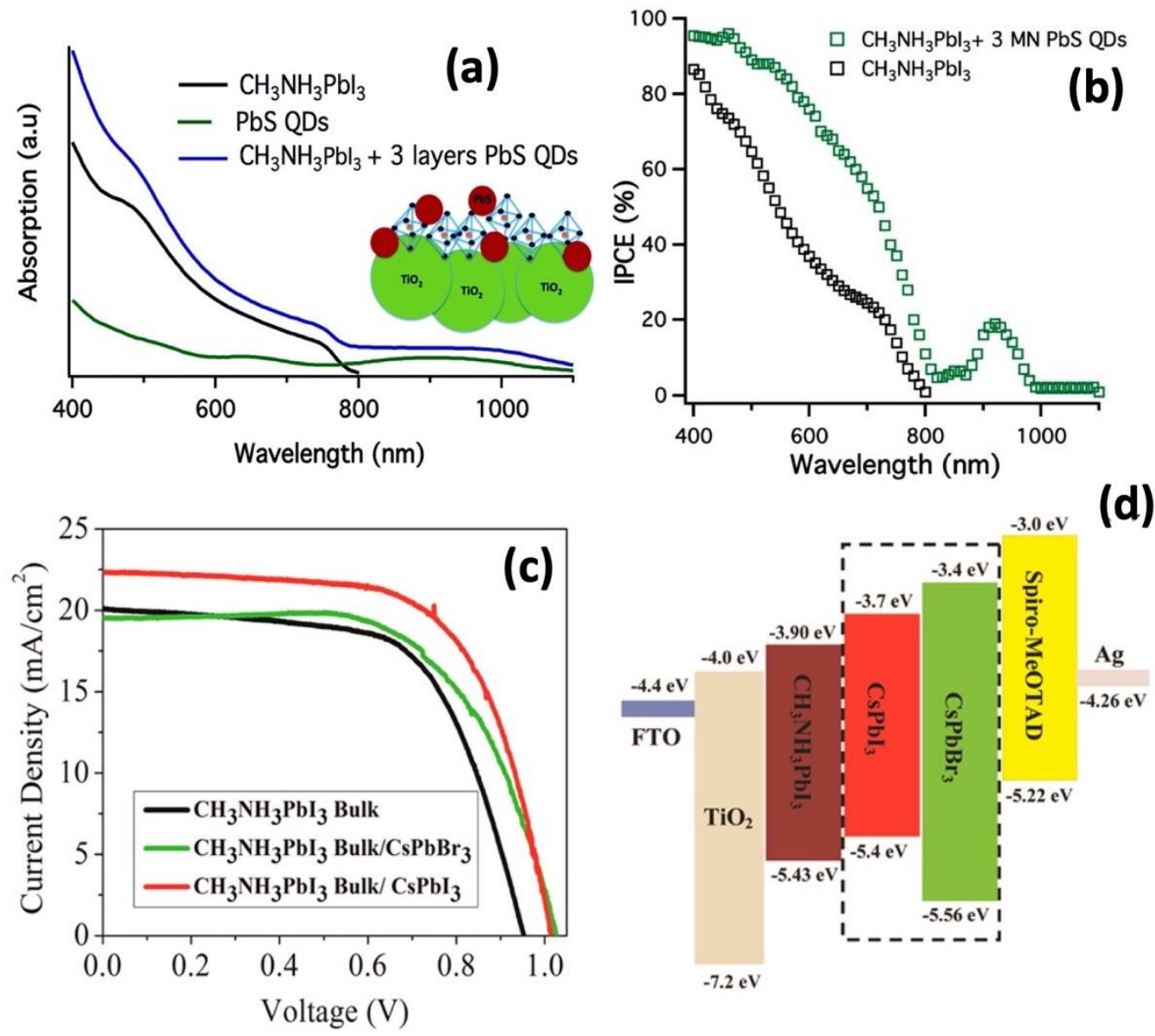

Figure 1: (a) Absorption spectra on mesoporous $\mathrm{TiO}_{2}$ substrate of the $\mathrm{PbS}$ QDs, lead iodide perovskite and lead iodide perovskite with 3 layers of $\mathrm{PbS}$ QDs. (b) Incident photon to current efficiency (IPCE) spectra of the PbS QDs, lead iodide perovskite and lead iodide perovskite with 3 layers of PbS QDs. Reproduced with permission of the Royal Society of Chemistry. ${ }^{44}$ (c) J-V curves spectra of perovskite solar cells incorporating $\mathrm{CsPbX}_{3}$ QDs between the $\mathrm{MAPbI}_{3}$ active layer and the HTM (d) Energy band diagram of each material within the perovskite/CsPbX 3 structure, with energy levels given in $\mathrm{eV}$. Reproduced with permission of the American Chemical Society. ${ }^{55}$

Interface Engineering in PSCs using perovskite QD layer sandwiched between the perovskite active layer and the HTM has produced a performance enhancement. ${ }^{55-57}$ Cha et al. reported an increase of PSC photoconversion efficiency by the deposition of $\mathrm{MAPbBr}_{0.9} \mathrm{I}_{2.1}$ between $\mathrm{MAPbI}_{3}$ and spiroOMeTAD HTM Quantum Dots. ${ }^{57}$ Moreover, inorganic perovskite QDs present higher stability as Sidhik et al. pointed out. ${ }^{55}$ The thin layer of $\mathrm{HP}\left(\mathrm{CsPbX}_{3}, \mathrm{X}\right.$ is $\mathrm{Br}$ or I) QDs deposited on top a $\mathrm{MAPbI}_{3}$ layer played acted as the electron blocking of 
the perovskite/HTM interface, reducing interfacial recombination and increasing consequently the open circuit potential, see Figure 1c. With an appropriated band alignment, using $\mathrm{CsPbI}_{3}$ QDs (see Figure 1d), the hole injection is not hindered and the photocurrent can be also enhanced, see Figure 1c. ${ }^{55}$ There are some other related studies regarding the interaction of perovskite QD with perovskite thin layers that have been recently summarized in a perspective article by Yang et al. ${ }^{56}$

On the other hand, one of the key issues on the efficiency improvement experienced in the last decade in colloidal QD solar cells, both on depleted heterojunction ${ }^{58}$ and in sensitized configuration, ${ }^{40}$ has been the passivation of surface trap states of colloidal QDs. HPs also exhibit good performance as passivation agents of QDs surface. Typically, colloidal QDs are synthesized with long-chain organic ligands which stabilize, control size and afford an excellent monodispersity. However, the bulky organic ligands create electronic barriers that compromise efficient charge transport of QD films in optoelectronic devices. To build a high-quality QD-based photovoltaic device, interdot electronic communication needs to be improved. On the other hand, organic ligands also act as surface passivators. QDs possess an inherent large surface-to-volume ratio, resulting in unsaturated dangling bonds, creating undesired electronic trap states within the bandgap of QDs. These trap states increase the chance of carrier recombination, curtailing the efficiency of charge extraction. The ligand exchange procedure opens the possibility to exchange to short ligand, improving the interdot electronic communication, and moreover passivate the trap states. Among various ligand exchange schemes, HPs, including HP precursors, shows a strong passivation and well-protection from oxygen, resulting an improvement in a device performance and stability.

There are several examples of exchange of bulky organic capping layer of colloidal QDs by HPs or HPs precursor shell. In 2014, Dirin et al. presented a broad study of the use of HPs and other metal halide complexes as inorganic capping ligands for colloidal nanocrystals ( $\mathrm{PbS}, \mathrm{CdSe}, \mathrm{CdS}, \mathrm{CdSe} / \mathrm{CdS}, \mathrm{InP}, \mathrm{Au}$ and $\mathrm{Pd}){ }^{59}$ showing that $\mathrm{MAPbI}_{3}$ was the first example of hybrid capping of $\mathrm{PbS}$ able to retain highly efficient IR photoluminescence quantum yield (PLQY) of PbS QDs. In the same year, Ning et al. carried out the solution-phase ligand exchange from the oleic acid capping of $\mathrm{PbS}$ QDs to methylammonium iodide (MAI). ${ }^{60}$ Short-ligand exchanged PbS QDs could be directly deposited in a single step, with no requirement of solid-state exchange, rendering it compatible with roll-to-roll processes such as screen printing, spray coating, and blade coating. Moreover MAI capped $\mathrm{PbS}$ QD films provided a good stability at $100^{\circ} \mathrm{C}$ annealing temperature. These QD films with MAI short-capping were applied to fabricate QD solar cells, reaching a photovoltaic conversion efficiency of $6.10 \% .{ }^{60}$

Next year, taking advantage of the similar crystal structure and lattice match between $\mathrm{PbS}$ and $\mathrm{MAPbI}_{3}$ Yang et al. used $\mathrm{MAPbI}_{3}$ to exchange the ligand of $\mathrm{PbS}$ QDs, see the Figure $2 \mathrm{a}^{46}$ High resolution transmission electron microscopy (HRTEM) measurements and the analysis of lattice fringes from scanning tunneling electron microscope (STEM) pointed out the core-shell structure of $\mathrm{PbS} / \mathrm{MAPbI}_{3}$ QDs after ligand exchange. An annealing is necessary to transform HP precursors 
into thin crystalline matrix to archive good solar cell efficiency. By introducing the coherently lattice-matching thin shell of $\mathrm{MAPbI}_{3}$, QDs were passivated, limiting the aggregation and thus achieving a better balance of open circuit potential, $V_{o c}$, and $J_{s c}$, yielding improved device performance, reaching $8.95 \%$ of photoconversion efficiency, using the depleted heterojunction quantum dot solar cell configuration. 46 The main mechanism in the ligand exchange from organic QD capping to MAI or $\mathrm{MAPbI}_{3}$ shell is based on the binding of iodine anions to lead atoms on the QD surface ${ }^{60}$ However, agglomeration could be produced. With a proper optimization of the ligand exchange process the inclusion of iodine on QD surface was done without the detrimental effect of fusion. ${ }^{61}$ A co-solvent system was employed to tune the solvent polarity in order to achieve the solvation of MAI and the dispersion of hydrophobic PbS QDs simultaneously in a homogeneous phase enhancing the performance of QD solar cells to $10.6 \% .^{61}$ This strategy has been further improved using lead halide passivators reaching a solar cell efficiency of $11.28 \%{ }^{62}$ Moreover the passivation of lead halide also improved the stability of devices, $90 \%$ of initial efficiency was maintained after 1000 hours stored in ambient conditions. ${ }^{62}$

$\mathrm{MAPbI}_{3}$ shell showed an excellent passivation of PbS QDs however QD solar cells strongly suffer from hysteresis in photocurrent-voltage curves. ${ }^{46}$ An interesting alternative to hybrid halide perovskite is the use of inorganic halide perovskites as $\mathrm{CsPbI}_{3}$, that exhibits higher stability. ${ }^{63}$ Although, perovskite $\alpha$-phase is not stable for bulk $\mathrm{CsPbI}_{3}$, it can be stabilized in $\mathrm{CsPbI}_{3}$ QDs. ${ }^{64}$ On the other hand, it also possesses similar crystal structure and low lattice mismatch (less than $5 \%$ ) comparing with those of $\mathrm{PbS}^{52}$ Very recently, Zhang et al. used $\mathrm{CsPbI}_{3}$ passivated $\mathrm{PbS}$ QDs. ${ }^{65}$ As in the case of $\mathrm{MAPbI}_{3}$, after ligand exchange from organic capping to $\mathrm{CsPbI}_{3}$, a $\mathrm{PbS} / \mathrm{CsPbI}$ core-shell QD structure also formed. $\mathrm{CsPbI}_{3}$ shell passivates the $\mathrm{PbS}$ QD surface, diminishing the trap states, hence improving the charge collection and reducing the charge recombination rate, allowing to obtain performances as high as $10.5 \%$. In addition, colloidal QD solar cells based on $\mathrm{PbS} / \mathrm{CsPbI}_{3}$ QDs present extremely low hysteresis, see Figure $2 \mathrm{~b}$, not only in the different scanning directions but also in different scan sweep steps. Moreover these devices also show a good stability not only under long illumination, 46 hours at continuously one sun illumination, see Figure $2 \mathrm{c}$, but also under ambient conditions (42 days). ${ }^{65}$

Perovskite passivation of $\mathrm{PbS}$ QDs have been also used successfully in QD sensitized solar cell configuration. ${ }^{45},{ }^{6} \mathrm{MAPbI}_{3}$ layer coated on $\mathrm{PbS} \mathrm{QDs}$ in sensitized solar cells, passivates QD surface, reducing significantly the defects as the $\mathrm{PbSO}_{3}$ oxidation, resulting finally in a better charge extraction with an improvement of the performance of the photovoltaic device. 

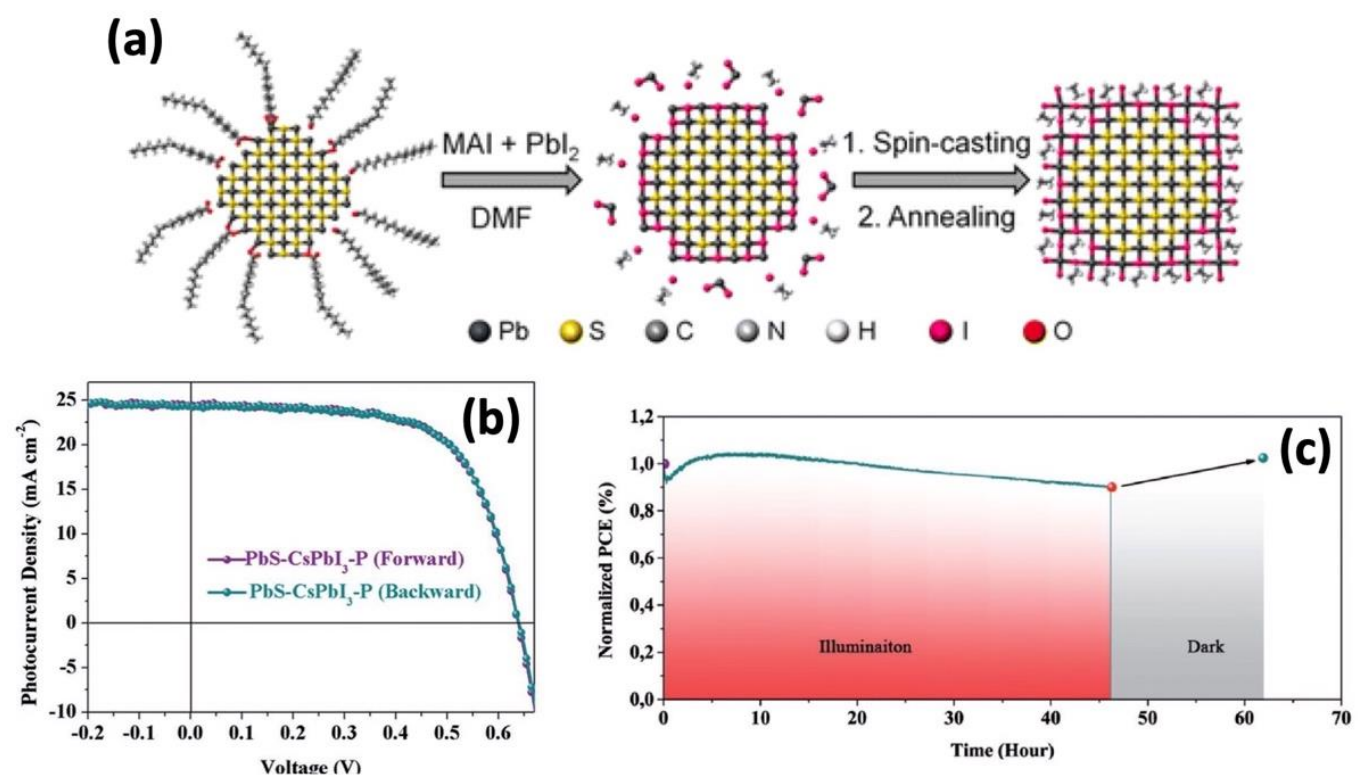

Figure 2: (a) Schematic of solution ligand exchange with $\mathrm{MAPbI}_{3}$ perovskite precursors and corresponding colloidal QD film preparation. Reproduced with permission of the American Chemical Society. ${ }^{46}$ (b) Forward and backward $J-V$ curves under 1 sun AM1.5G illumination of $\mathrm{PbS} / \mathrm{CsPbI}{ }_{3}$ based colloidal QD solar cell. (c) Photostability test of the $\mathrm{PbS} / \mathrm{CsPbI}{ }_{3}$ based QD solar cell under illumination and after stored during $16 \mathrm{~h}$ in ambient conditions under dark after the illumination test. The photostability test was performed in $\mathrm{N}_{2}$ atmosphere under one sun equivalent illumination provided by a white LED lamp. Reproduced with permission of Wiley. ${ }^{65}$

Nevertheless, beyond of the use of halide perovskite as mere passivation agent of colloidal QDs, likely the most appealing results have been obtained when a more synergistic interaction was stablished between both materials. In 2015, Ning et al. reported by first time the preparation of thin films with perovskite matrix and embedded colloidal QDs. ${ }^{24} \mathrm{PbS}$ and $\mathrm{MAPbI}_{3}$ possess a related crystal structure, each having a six-coordinated $\mathrm{Pb}$ atom, and with $\mathrm{Pb}-\mathrm{Pb}$ distances $\left(\mathrm{MAPbI}_{3}\right.$, $6.26 \mathrm{~A}^{\circ}$; $\mathrm{PbS}, 5.97 \mathrm{~A}^{\circ}, \sim 5 \%$ lattice mismatch), see Figure 3a-b. Their analysis of density functional theory (DFT) of an interface formation between $\mathrm{PbS}$ (100) and $\mathrm{MAPbI}_{3}(110)$ plane showed an interfacial energy less than $10 \mathrm{meV} \cdot \AA^{-2}$, suggesting that growth of $\mathrm{HP}$ on $\mathrm{PbS}$ at room temperature is nearly as feasible as homoepitaxy of $\mathrm{PbS}$ on $\mathrm{PbS}$ or HP on HP. DFT further reveals that the epitaxial threedimensional embedding of PbS CQDs inside a HP matrix is achieved without the formation of interfacial defects; that is, the bandgap is predicted to remain open, with no in-gap defects predicted. They concluded structurally PbS QDs lattice has a relative good match with $\mathrm{MAPbI}_{3} \mathrm{HP}$ structure both three dimensionally and two dimensionally. ${ }^{24}$ Perovskite matrix with embedded QDs increased significantly the $\mathrm{PL}$ emission from $\mathrm{PbS}$ QDs as photogenerated electrons and holes in HP were transferred to $\mathrm{PbS}$ QDs with a value exceeding $80 \%$ of efficiency. Since this first demonstration, HP films with embedded QDs were applied as an active layer in different optoelectronic devices such as solar cells ${ }^{67-68}, \operatorname{LEDs}^{26,} 48$ and 
photodetectors. ${ }^{49-51}$ In these works, embedding QDs inside HPs matrix showed an improvement respect to the reference devices, without embedded QDs.

(a)

(b)
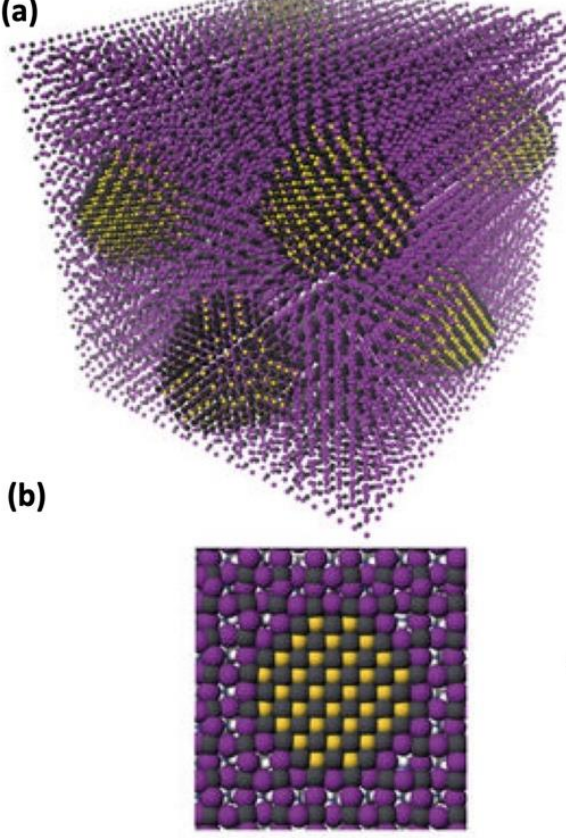

(c)

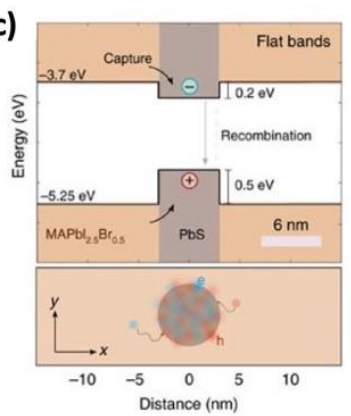

(e)

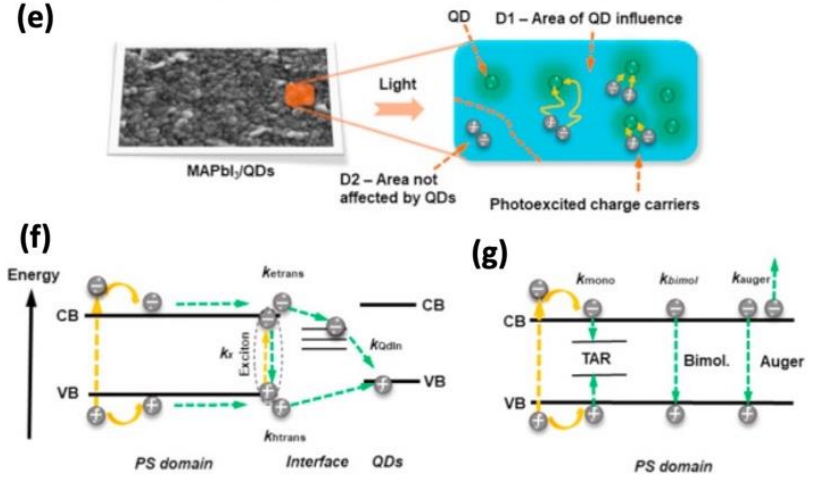

(f)

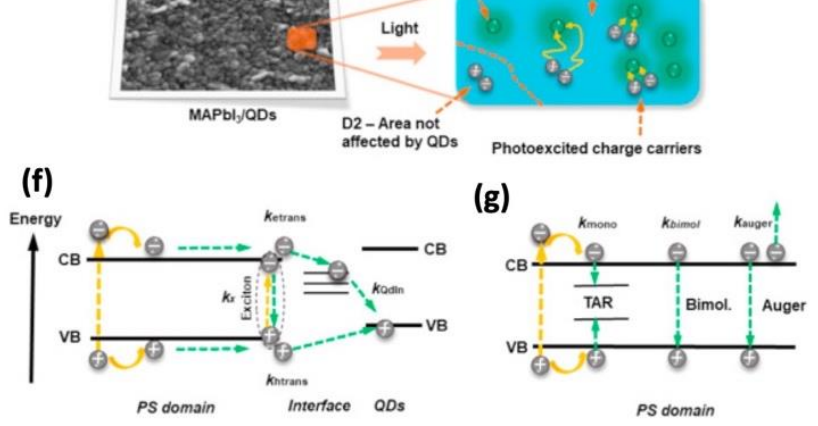

(d)

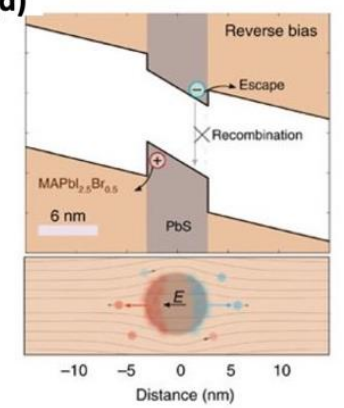

$Q D \quad D 1-$ Area of $Q D$ influence

Figure 3: (a) Three-dimensional atomistic model and (b) Cross-section (two-dimensional view) of $\mathrm{PbS}$ QDs embedded in a halide perovskite matrix. (a-b) Reproduced with permission of Nature. ${ }^{24}$ (c, d) Band alignment of PbS QDs embedded in mixed halide perovskite matrix as calculated with a SCAPS model at (c) no bias and (d) reverse bias conditions. In the absence of an imposed electric field photogenerated charges are uniformly distributed across the dot (bottom panels). At sufficient reverse bias conditions they are spatially separated and can inject into the $\mathrm{MAPbI}_{2.5} \mathrm{Br}_{0.5}$ host, favoring carrier recirculation over capturing. (c-d) Reproduced with permission of Nature. ${ }^{50}$ (e) $\mathrm{SEM}$ of $\mathrm{MAPbI}_{3}$ layer with embedded $\mathrm{PbS} / \mathrm{CdS}$ core/shell QDs and the cartoon showing the areas of QDs influence (D1) and without their influence (D2) upon excitation over the band gap of perovskite. (f) Energy diagram showing the dynamics of photoexcited charge carriers in $\mathrm{MAPbI}_{3^{-}}$ QDs film in D1 area and (g) D2 area upon excitation over the band gap. For clarity, (f) only shows the processes involving the influence of QDs, while those in $(\mathbf{g})$ representing the classical events in pure perovskite. $k_{\text {etrans }}$ and $\mathrm{k}_{\mathrm{htrans}}$ characterize the effective transfer processes of charge carriers from perovskite to the interface or/and QDs. $\mathrm{k}_{\mathrm{x}}$ is the exciton recombination rate constant. $\mathrm{k}_{\mathrm{QdIn}}$ is the recombination rate constant between trapped electrons and holes in QDs. (e-g) Reproduced with permission from Elsevier. ${ }^{69}$

The introduction of $\mathrm{PbS}$ QDs into HP matrix has increased photoconversion performance of PSCs by the effect of QDs in the crystallization process of perovskite layer. $\mathrm{Li}$ et al. prepared solar cells with $\mathrm{MAPbI}_{3-\mathrm{x}} \mathrm{Cl}_{\mathrm{x}}$ matrix an embedded PbS QDs by dispersing a small quantity of core/shell PbS/MAI QDs in the solution containing the HP precursors. PbS acted as effective seed-like sites to promote the formation of HP grains, resulting in substantial morphological improvements in terms of grain size, surface coverage and uniformity. Similar 
effect in the grain size by QD addition have been also observed in other works. ${ }^{26}$ The PSC performance observed by the appropriate incorporation of $\mathrm{PbS}$, with concentration $1.0 \mathrm{wt} \%$, reached $17.4 \%$, proximately a $25 \%$ of improvement respect the reference devices. ${ }^{67}$ Recently, Han et al. attained a performance of $18.6 \%$ with the QD in perovskite strategy. ${ }^{68}$

In this sense, the maximum photoconversion efficiency with hybrid QD and HP films have been obtained with QDs acting with a passive role, as additives in order to obtain a better perovskite crystallization. This is not the case in hybrid QDHP LEDs where the QDs are the active light emitters and HPs act as carrier transporting matrix. Gong et al. reduced the lattice mismatch between $\mathrm{HP}$ and $\mathrm{PbS}$ QDs (with exciton peak at $1391 \mathrm{~nm}$ ) by incorporating a small quantity (17\%) of bromide into $\mathrm{MAPbI}_{3}$ matrix, forming a mixed halide $\mathrm{MAPbI}_{3-\mathrm{x}} \mathrm{Br}_{\mathrm{x}}{ }^{48}$ The introduction of bromide reduced on one hand the strain at the interface of HP and QDs, and on the other hand the amount of defect on $\mathrm{PbS}$ surface. The function of a mixed halide perovskite is to transport generated charger carriers into $\mathrm{PbS}$ QDs. The selected HP and PbS QDs presented a type I band alignment, see Figure 3c, and the transported carriers in the perovskite matrix are finally injected into the $\mathrm{PbS}$ QDs where they recombine radiatively, resulting in an increase of the LED external quantum efficiency (EQE), reaching 4.9\%, two folds higher than pure QDs devices. ${ }^{48}$ This approach has been also used by the same group but with layered $2 \mathrm{D}$ perovskites, incorporating longer amine molecules, butylamine, pentylamine and hexylamine. The substitution of $\mathrm{MA}^{+}$cation by these amine molecules with longer carbon backbones breaks the symmetry of the 3D structure and introduces 2D structuring layered along c-axis. The QDs exchanged were easily deposited in a single step. By using 2D HP passivated PbS QDs as an active layer, the authors could increase the operating voltages of LEDs up to $7.5 \mathrm{~V}$ due to better stability of $2 \mathrm{D} \mathrm{HPs}$ in a comparison with $3 \mathrm{D} \mathrm{HPs.}{ }^{47}$

Embedding QDs inside HPs matrix was applied in photodetectors as well. Liu et al. pointed out that the incorporation of $\mathrm{PbS}$ QDs induced traps-assisted charge injection. ${ }^{49} \mathrm{PbS}$ QDs produced traps residing on the surface of $\mathrm{MAPbI}_{3}$ thin films. Under illumination, excitons formed in HP layer rapidly would dissociate into electrons and holes. Then, electrons would be trapped at the HP-PbS QD surface which would shift downward the conduction band (CB) energy level of HPQD nanocomposites, followed by the re-alignment of its fermi level with the work function of the $\mathrm{Al}$ cathode. The trapped electrons can be considered as a heavydoping on the surface of HP-QDs layer, which dramatically narrow the depletion region formed at the interface. Thus injected holes from Al cathode can be easily tunneled through the hole blocking layers (PC61BM/BCP) at low external biases, resulting an EQE of $\sim 4500 \%$, a photoresponsivity of $\sim 15,000 \mathrm{~mA} / \mathrm{W}$ and a detectivity of over $6 \times 10^{13}$ Jones $\left(1\right.$ Jones $\left.=1 \mathrm{~cm} \cdot \mathrm{Hz}^{1 / 2} \cdot \mathrm{W}^{-1}\right)$ at a small bias of $2 \mathrm{~V}$, and a photoresponse time of $11.5 \mu \mathrm{s} .{ }^{49}$

Moreover, following the strategy of lattice matching developed by Gong et al. ${ }^{48}$ a further study on the interaction between HP and PbS QDs under applied reverse bias condition for the development of photodetectors has been performed 
by the same group. ${ }^{50}$ The lattice of mixed HP was adjusted, by optimizing the ratio between iodide and bromide $\mathrm{MAPbI}_{2.5} \mathrm{Br}_{0.5}$ to suitable to that of $\mathrm{PbS}$ QDs (exciton peak at $1240 \mathrm{~nm}, \sim 1 \mathrm{eV}$ ). The photodiode has been prepared using a structure of FTO/compact $\mathrm{TiO}_{2} / \mathrm{PbS}$ QDs embedded in $\mathrm{HP}$ matrix/spiro-OMeTAD/Au. Under no external electrical field, generated excitons are confined inside QDs due to the type I junction forming between HP and QDs, and lose their energy via the recombination, radiative or non-radiative, see Figure 3c. However, under the presence of a suitably large electric field, Figure $3 \mathrm{~d}$, the excitons photogenerated in the QDs will then separate, and the charges may be ejected from the dots, under the action of the field, into the host perovskite matrix. Escape charge carriers can be potentially re-captured into QDs, as determined by the competition between carrier thermalization and extraction time. This structure allowed the preparation of photodetectors with specific detectivities that exceed $10^{12}$ Jones. $^{50}$

In order to further improve the performance of optoelectronic devices developed with HP-QD systems, the understating of the deep interaction of these materials is mandatory. Recently, we have furthermore elucidated the interaction mechanism between HP and QDs. We studied the charge carrier dynamics of HPQDs hybrid films as function of QD concentrations ( $\left.\mathrm{C}_{\mathrm{QDs}}\right)$ using ultrafast UV-VIS transient absorption (TA) and $\mathrm{THz}$ spectroscopies measurements. ${ }^{69}$ By photogenerating charge carriers over the band gap of $\mathrm{MAPbI}_{3}(\sim 1.55 \mathrm{eV})$ in the HP-QDs hybrid material, we observed fast and efficient charge carrier deactivation due to their transfer to the QDs. The TA decay becomes shorter upon increasing $\mathrm{C}_{\mathrm{QDs}}$. For samples containing $\mathrm{C}_{\mathrm{QDs}}$ lower than $0.18 \mathrm{wt} \%$, the $\mathrm{TA}$ decays were governed by the behavior of neat HP while for higher concentrations a progressive faster decay was observed. ${ }^{69}$ The observed dynamics were analyzed in terms of two different domains of the HP layer: a domain D1 where photocarriers are influenced by the presence of QDs and a domain D2 where they do not interact with QDs. The events happened in D1 were described in Figure 3f: (i) charge carriers transport and transfer from $\mathrm{HP}$ domain to QDs and $\mathrm{MAPbI}_{3} / \mathrm{QDs}$ interface, (ii) exciton recombination, and (iii) recombination of transferred charge carriers. In addition, electron and hole recombination processes in HP is also produced. Using a kinetic model and analyzing the TA signals, we observed that $85-89 \%$ of both electrons and holes were transferred to the HP/QDs interface trap states, and to the valence band (VB) of the QDs. Up to 7\% charges formed excitons in HP domain in the close vicinity of interface. A small fraction of photoexcited charges $(8-15 \%)$ were not affected by the presence of QDs, and thus, their deactivation followed the standard processes of neat HP, see Figure $3 g$. The changes in the values are related to the QDs concentration in the matrix. The analysis of the data shows the important role of trap states and gives clues for the further improvement of this hybrid system. The size of D1 and D2 domains depends on the QD concentration and on the carrier diffusion length in HP, consequently this method can be also used to determine the diffusion length of the HP layer as we showed. ${ }^{69}$

The interaction of HP and QDs has been studied not only for hybrid organicinorganic HP but also with a fully inorganic HP. Jung et al. pointed out that $\mathrm{PbS}$ 
QDs and $\mathrm{CsPbBr}_{3}$ present just $~ 0.5 \%$ lattice mismatch, much lower than the lattice mismatch between $\mathrm{PbS}$ and $\mathrm{MAPbI}_{3}, \sim 5 \%{ }^{52}$ They studied theoretically the bond formation and carrier confinement at the HP-QD interface. By calculating the surface energy at different facets of $\mathrm{PbS}$ and $\mathrm{CsPbBr}_{3}$, they could construct the interface slab models along (100) orientation. Using the models and calculating the interface energy taking into account different terminations, the growth mechanism of HP-QD interface is elucidated. (100) facet of $\mathrm{CsPbBr}_{3}$ with a termination of $\mathrm{PbBr}_{2}$ spontaneously grows on the (100) facet of $\mathrm{PbS}$ by locating $\mathrm{Pb}$ on $\mathrm{S}$ and $\mathrm{Br}$ on $\mathrm{Pb}$ with the presence of a strong chemical interaction attributed to heteropolar bond formation. 52

Finally, combined systems of HP and colloidal QDs have also demonstrated the potentiality for the development of advanced optoelectronic devices, not by the enhancement of the properties already presented in HP or colloidal QDs but by presenting new ones. Single layers and bilayers of $\mathrm{MAPbI}_{3}$ and colloidal core/shell $\mathrm{PbS} / \mathrm{CdS}$ QDs were prepared, using two different QD sizes, with diameter 2.3 and $3 \mathrm{~nm} .{ }^{25}$ Figure 4 a shows the normalized photoluminescence (PL) of the single HP and QD layers. In addition, bilayers formed by both combinations of QD/HP and HP/QD were also studied with a type II alignment due to the relative large bandgap of the $\mathrm{PbS} \mathrm{QDs}$ in comparison with other experiments. Interestingly bilayer samples presents a new feature in the near infrared region (NIR) that it is not observed for single layer samples, HP or QD, as can be observed in Figure 4b. In this region perovskite single layer does not present any PL while for single layer colloidal QD sample just the queue of the band edge emission is observed. However bilayers presents an emission at 1200-1600 range blue shifted for QDs with smaller size, see Figure $4 \mathrm{~b}$. The intensity of this emission increases when samples are excited with wavelengths shorter than the perovskite bandgap and it is attributed to the formation of an exciplex state between HP and QD. ${ }^{25}$ The exciplex emission has been also observed in perovskite films with embedded QDs. ${ }^{26}$

The use of different emitters produced LEDs with color tuned by the applied bias, see Figure 4c. The color can be easily controlled as the ratio between QD and HP EL intensity emission follows a linear relationship, see Figure 4d. Interestingly, the emission from HP-QD exciplex state is observed not only by optical pumping, Figure $4 \mathrm{~b}$, but also by electrical injection, Figure $4 \mathrm{e}$, with a voltage threshold as low as $0.77 \mathrm{~V}$, see Figure $4 \mathrm{f}^{25}$ The possibility of band gap tuning of the HP by the use of different cations and anions and of the colloidal QDs can potentially develop dual LEDs working with very different wavelength depending of the applied bias.

Moreover the combination of HP and colloidal QDs can have also an interesting potentiality for the development of advanced photovoltaic devices. Due to the reciprocity principle, if an electron and hole from an exciplex state can recombine producing a photon, the reciprocal process in which a photon can produce an electron-hole pair at the exciplex state is also possible. This possibility opens the door to the preparation of Intermediate Band Gap Solar Cells (IBSCs) taking benefit from the HP and QD synergies. 
The concept of IBSC was developed by Luque and Martí. ${ }^{70}$ In an IBSC there are one or several intermediate bands at the band gap of a wide gap semiconductor, been $E_{G}$ the band gap energy, see Figure $4 \mathrm{~g}$, for the case of just one intermediate band. Photons with energy higher than $E_{G}$ can be absorbed by the matrix semiconductor, process 1 in Figure 4g. Moreover, intermediate bands would act as stepping stones allowing low energy photons transfer electrons from the VB of the wide band gap semiconductor to its $\mathrm{CB}$ by the absorption of a couple of photons with energies $E_{H}$ and $E_{L}$, processes 2 and 3 in Figure $4 \mathrm{~g}$. In order to take advantage of the intermediate band this level has to be isolated from the extracting contact and three quasi Fermi levels can be defined for VB, CB and intermediate band. ${ }^{70-71}$ This fact allow that the IBSC retains the $V_{o c}$ of the wide band gap semiconductor defined by the splitting of electrons in $\mathrm{CB}$ and holes in VB Fermi levels, $E_{F n}$ and $E_{F h}$ respectively, but with higher photocurrent due to the sub-band gap photon absorption, see Figure 4g. In an optimum situation of an IBSC, a half filled intermediate bandgap can either receive electrons from the VB of the matrix compound (process 2 in Figure $4 \mathrm{~g}$ ) and promote electrons from the intermediate band state into the $\mathrm{CB}$ of the matrix (process 3 in Figure $4 \mathrm{~g}$ ). Theoretical calculations predicts a maximum photoconversion efficiency of $63 \%$ under high solar concentration for an cell with an optimum IB state in contrast with a maximum of $41 \%$ predicted for single absorber by the Shockley-Queisser limit at the same conditions, ${ }^{70}$ with a performance higher than an optimized tandem cell formed by a couple of absorbers. IBSC will have higher theoretical quantum efficiency than tandem cells at any illumination condition. A tandem cell has a maximum quantum efficiency of 0.5 in all the spectra, needing two photons to extract one electron. In the case of IBSCs the same situations accounts for photons with energy lower than $E_{G}$, whereas for photons with higher energy quantum efficiency of 1 is possible.
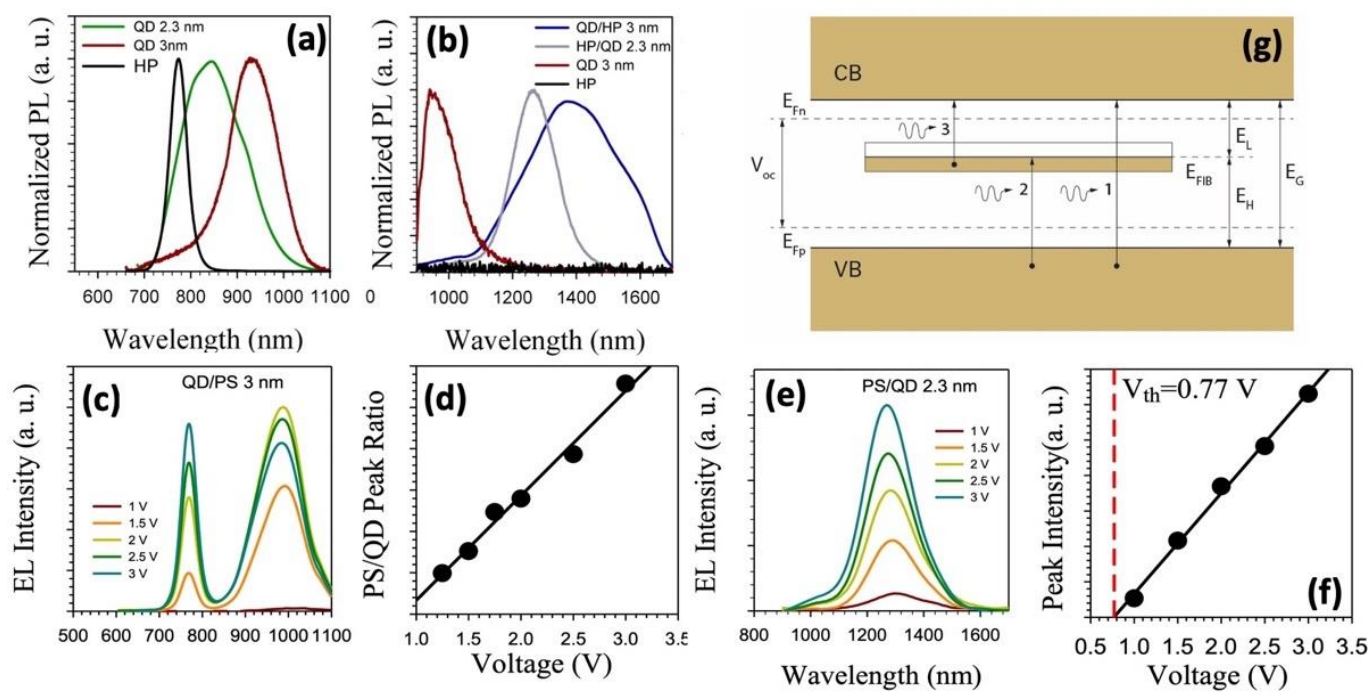

Figure 4: (a) PL of single layers of $\mathrm{MAPbI}_{3}$, and core/shell $\mathrm{PbS} / \mathrm{CdS}$ QDs 2.3 and $3 \mathrm{~nm}$ diameter size corresponding to the band gap transition. (b) PL in the NIR (900-1700 nm) showing a new exciplex transitions in HP-QD bilayers while no new peak is observed in for single HP or QD (for 
QD layer just the queue of the band gap transition). (c) EL at different applied bias of a bilayer sample of $\mathrm{HP}$ and $\mathrm{PbS} / \mathrm{CdS}$ QDs of $3 \mathrm{~nm}$. (d) Ratio between the EL intensity peak of PbS/CdS QDs and HP at different applied bias. (e) EL at different applied bias in the NIR region of a bilayer sample of $\mathrm{HP}$ and PbS/CdS QDs of $3 \mathrm{~nm}$. (f) EL intensity peak in the NIR of PbS/CdS QDs 3nm and HP at different applied bias. (g) Scheme of energy configuration of an Intermediate Band Gap Solar Cell. (a-g) Reproduced with permission of AAAS. ${ }^{25}$

However, the development of an IBSC with significant efficiency has been elusive. For example the use a monocrystalline GaAs host matrix and InAs QDs grown in the Stranski-Krastanov mode, presented severe limitations in order to produce a practical solar cell. The photocurrent increase is limited by the low QD density. ${ }^{72}$ The density of QDs cannot be very high as QDs produce strain in the layer, and very high strain causes the formation of defects that degrades severely the cell performance. ${ }^{71}$ However, the use of HP could help to solve these problems as strain is avoided as it is relaxed at the benign perovskite grain boundaries. Moreover, both HPs and QDs present a high versatility allowing tailoring the intermediate band and the band alignment between HP and colloidal QDs to produce an optimum IBSC.

In summary, the interaction between HP and colloidal QDs can produce interesting constructing synergies promoting the properties of each material and generating new ones. Each material obtains benefits from the interaction with the other, HP can increase the passivation of the QD surface, protecting them from the photocorrosion. Moreover, HP can benefit from the appropriate use of QDs in the selective contacts. HP matrix can also work as an excellent carrier transporter into QDs in a view of type I junction for the development of LEDs and photodetectors while QDs acted as effective seed-like effect to promote the proper crystallization of HP lattice structures, resulting in substantial morphological improvements. Furthermore the band alignment between HP and QDs can be tailored to favor the injection of photogenerated electrons and holes from one into the other. Hence this combination could lead to an improvement in performance of optoelectronic devices such as solar cells and LEDs. On the other hand, the interaction between HP and colloidal QDs also rises new properties, which do not exist in singles materials, as the formation of the exciplex state at lower energies than both HP and QD band gap. ${ }^{25-26}$ This system presents a high potentiality in order to develop advanced optoelectronic devices as tunable color LED or intermediate band gap solar cells. However, non-radiative recombination has to be avoided as a major challenge of these advanced configurations in order to get a full benefit of this potentiality. Further study on the interaction between these families of materials undoubtedly will contribute to a further optimization of current devices and to explore new and exciting constructive synergies.

\section{AUTHOR INFORMATION \\ Corresponding Author}

*Address correspondence to this author: sero@uji.es Twitter: @IvanMoraSero Notes 
The authors declare no competing financial interest.

\section{ACKNOWLEDGMENT}

This work was supported by European Research Council (ERC) via Consolidator Grant (724424 - No-LIMIT) and by the Generalitat Valenciana via project Prometeo/2018/098 (Q-Devices).

\section{Biographies}

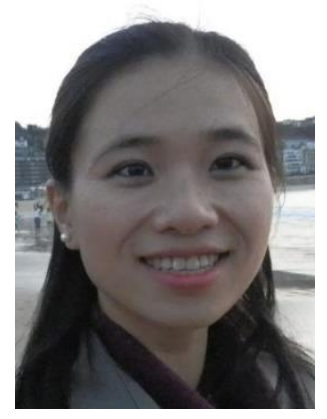

Thi Tuyen Ngo graduated in Physics from Vietnam National University (Vietnam) in 2008 and received her Master from University of the Basque Country (Spain) in 2011. She is currently a Ph.D. student at the Institute of Advanced Materials (INAM), Jaume I University (Spain). Her study is focusing on the interaction of perovskite and other materials such as quantum dots and organic compounds. Before incorporating in a Ph.D, she worked as assistant at Institute of Physics, Vietnam Academy Science and Technology (2008-2009) and worked in the photovoltaic cells at Cidetec (2011-2013) where she learnt about the preparation and characterization of solar cells.

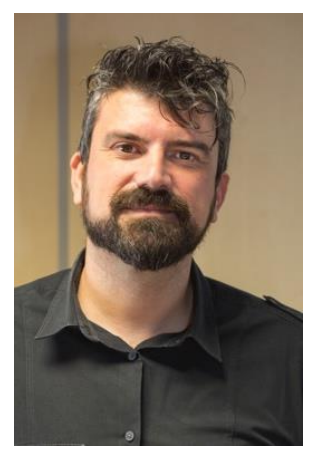

Iván Mora-Seró is associated professor at the Institut of Advanced Materials (INAM) at Universitat Jaume I de Castelló. His research has been focused on crystal growth, nanostructured devices, transport and recombination properties, photocatalysis, electrical characterization of photovoltaic, electrochromic, and water splitting systems, making both experimental and theoretical work. Recent 
research activity is focused on new concepts for photovoltaic conversion and light emission (LEDs and light amplifiers) based on nanoscaled devices and semiconductor materials following two mean lines: semiconductor quantum dots and lead halide perovskites. He has appear in the list of Highly Cited Researcher of Clarivate Analytics the three last years.

\section{References}

1. NREL photovoltaic efficiency chart: https://www.nrel.gov/pv/assets/pdfs/pvefficiency-chart.20181221.pdf

2. Dou, L.; Yang, Y.; You, J.; Hong, Z.; Chang, W.-H.; Li, G. Solution-processed hybrid perovskite photodetectors with high detectivity. Nat. Commun. 2014, 5, 5404.

3. Jaramillo-Quintero, O. A.; Sanchez, R. S.; Rincon, M.; Mora-Sero, I. Bright Visible-Infrared Light Emitting Diodes Based on Hybrid Halide Perovskite with SpiroOMeTAD as a Hole-Injecting Layer. J. Phys. Chem. Lett. 2015, 6, 1883-1890.

4. Tan, Z.-K. et. al. Bright light-emitting diodes based on organometal halide perovskite. Nat. Nanotechnol. 2014, 9, 687-692.

5. Alivisatos, A. P. Semiconductor Clusters, Nanocrystals, and Quantum Dots. Science 1996, 271, 933-937.

6. Lin, K. et. al. Perovskite light-emitting diodes with external quantum efficiency exceeding 20 per cent. Nature 2018, 562 (7726), 245-248.

7. Suárez, I.; Juárez-Pérez, E. J.; Bisquert, J.; Mora-Seró, I.; Martínez-Pastor, J. P. Polymer/Perovskite Amplifying Waveguides for Active Hybrid Silicon Photonics. Adv. Mater. 2015, 27, 6157-6162.

8. Suárez, I.; Hassanabadi, E.; Maulu, A.; Carlino, N.; Maestri, C. A.; Latifi, M.; Bettotti, P.; Mora-Seró, I.; Martínez-Pastor, J. P. Integrated Optical AmplifierPhotodetector on a Wearable Nanocellulose Substrate. Adv. Opt. Mater. 2018, 6, 1800201. 9. Deschler, F. et. al. High Photoluminescence Efficiency and Optically Pumped Lasing in Solution-Processed Mixed Halide Perovskite Semiconductors. J. Phys. Chem. Lett. 2014, 5, 1421-1426.

10. Zhu, H.; Fu, Y.; Meng, F.; Wu, X.; Gong, Z.; Ding, Q.; Gustafsson, M. V.; Trinh, M. T.; Jin, S.; Zhu, X. Y. Lead halide perovskite nanowire lasers with low lasing thresholds and high quality factors. Nat. Mater. 2015, 14, 636-642.

11. Xing, G.; Mathews, N.; Lim, S. S.; Yantara, N.; Liu, X.; Sabba, D.; Grätzel, M.; Mhaisalkar, S.; Sum, T. C. Low-temperature solution-processed wavelength-tunable perovskites for lasing. Nat. Mater. 2014, 13, 476-480.

12. Stranks, S. D.; Hoye, R. L. Z.; Di, D.; Friend, R. H.; Deschler, F. The Physics of Light Emission in Halide Perovskite Devices. Adv. Mater. 2018, 30, 1803336.

13. Mitzi, D. B. Synthesis, Structure, and Properties of Organic-Inorganic Perovskites and Related Materials. 1999, Progress in Inorganic Chemistry, Print ISBN: 9780471326236, DOI:10.1002/9780470166499.

14. Lee, M. M.; Teuscher, J.; Miyasaka, T.; Murakami, T. N.; Snaith, H. J. Efficient Hybrid Solar Cells Based on Meso-Superstructured Organometal Halide Perovskites. Science 2012, 338, 643-647.

15. Kim, H.-S. et. al. Lead Iodide Perovskite Sensitized All-Solid-State Submicron Thin Film Mesoscopic Solar Cell with Efficiency Exceeding 9\%. Sci. Rep. 2012, 2, 591. 
16. Jeon, N. J.; Noh, J. H.; Yang, W. S.; Kim, Y. C.; Ryu, S.; Seo, J.; Seok, S. I. Compositional engineering of perovskite materials for high-performance solar cells. Nature 2015, 517, 476-480.

17. Bi, D. et. al. Efficient luminescent solar cells based on tailored mixed-cation perovskites. Sci. Adv. 2016, 2, e1501170.

18. Yang, W. S. et. al. Iodide management in formamidinium-lead-halide-based perovskite layers for efficient solar cells. Science 2017, 356, 1376-1379.

19. De Wolf, S.; Holovsky, J.; Moon, S.-J.; Löper, P.; Niesen, B.; Ledinsky, M.; Haug, F.-J.; Yum, J.-H.; Ballif, C. Organometallic Halide Perovskites: Sharp Optical Absorption Edge and Its Relation to Photovoltaic Performance. J. Phys. Chem. Lett. 2014, 5, 10351039.

20. Etgar, L.; Gao, P.; Xue, Z.; Peng, Q.; Chandiran, A. K.; Liu, B.; Nazeeruddin, M. K.; Grätzel, M. Mesoscopic $\mathrm{CH}_{3} \mathrm{NH}_{3} \mathrm{PbI}_{3} / \mathrm{TiO}_{2}$ Heterojunction Solar Cells. J. Am. Chem. Soc., 2012, 134, 17396-17399.

21. Liu, M.; Johnston, M. B.; Snaith, H. J. Efficient planar heterojunction perovskite solar cells by vapour deposition. Nature 2013, 501, 395-398.

22. Kim, H.-S.; Mora-Sero, I.; Gonzalez-Pedro, V.; Fabregat-Santiago, F.; JuarezPerez, E. J.; Park, N.-G.; Bisquert, J. Mechanism of carrier accumulation in perovskite thinabsorber solar cells. Nat. Commun. 2013, 4, 2242

23. Ngo, T. T.; Suarez, I.; Antonicelli, G.; Cortizo-Lacalle, D.; Martinez-Pastor, J. P.; Mateo-Alonso, A.; Mora-Sero, I. Enhancement of the Performance of Perovskite Solar Cells, LEDs, and Optical Amplifiers by Anti-Solvent Additive Deposition. Adv. Mater. 2017, 29, 1604056.

24. Ning, Z.; Gong, X.; Comin, R.; Walters, G.; Fan, F.; Voznyy, O.; Yassitepe, E.; Buin, A.; Hoogland, S.; Sargent, E. H. Quantum-dot-in-perovskite solids. Nature 2015, 523, 324-328.

25. Sanchez, R. S.; de la Fuente, M. S.; Suarez, I.; Muñoz-Matutano, G.; MartinezPastor, J. P.; Mora-Sero, I. Tunable light emission by exciplex state formation between hybrid halide perovskite and core/shell quantum dots: Implications in advanced LEDs and photovoltaics. Sci. Adv. 2016, 2, e1501104.

26. Ngo, T. T.; Suarez, I.; Sanchez, R. S.; Martinez-Pastor, J. P.; Mora-Sero, I. Single step deposition of an interacting layer of a perovskite matrix with embedded quantum dots. Nanoscale 2016, 8, 14379-14383.

27. Shirasaki, Y.; Supran, G. J.; Bawendi, M. G.; Bulovic, V. Emergence of colloidal quantum-dot light-emitting technologies. Nat. Photon 2013, 7, 13-23.

28. Owen, J.; Brus, L. Chemical Synthesis and Luminescence Applications of Colloidal Semiconductor Quantum Dots. J. Am. Chem. Soc. 2017, 139,10939-10943.

29. Pal, B. N.; Ghosh, Y.; Brovelli, S.; Laocharoensuk, R.; Klimov, V. I.; Hollingsworth, J. A.; Htoon, H. 'Giant' CdSe/CdS core/shell nanocrystal quantum dots as efficient electroluminescent materials: strong influence of shell thickness on light-emitting diode performance. Nano Lett. 2012, 12, 331-6.

30. Sun, L.; Choi, J. J.; Stachnik, D.; Bartnik, A. C.; Hyun, B. R.; Malliaras, G. G.; Hanrath, T.; Wise, F. W. Bright infrared quantum-dot light-emitting diodes through interdot spacing control. Nat. Nanotechnol. 2012, 7, 369-73.

31. Wood, V.; Panzer, M. J.; Bozyigit, D.; Shirasaki, Y.; Rousseau, I.; Geyer, S.; Bawendi, M. G.; Bulovic, V. Electroluminescence from nanoscale materials via fielddriven ionization. Nano Lett. 2011, 11, 2927-32.

32. Lan, X. et. al. 10.6\% Certified Colloidal Quantum Dot Solar Cells via SolventPolarity-Engineered Halide Passivation. Nano Lett. 2016, 16, 4630-4. 
33. Im, J.-H.; Lee, C.-R.; Lee, J.-W.; Park, S.-W.; Park, N.-G. $6.5 \%$ efficient perovskite quantum-dot-sensitized solar cell. Nanoscale 2011, 3, 4088-4093.

34. Nozik, A. J. Quantum Dot Solar Cells. Physica E 2002, 14, 115-200.

35. Kongkanand, A.; Tvrdy, K.; Takechi, K.; Kuno, M.; Kamat, P. V. Quantum Dot Solar Cells. Tuning Photoresponse through Size and Shape Control of CdSe-TiO2 Architecture. J. Am. Chem. Soc. 2008, 130, 4007-4015.

36. Wang, J.; Li, Y.; Shen, Q.; Izuishi, T.; Pan, Z.; Zhao, K.; Zhong, X. Mn doped quantum dot sensitized solar cells with power conversion efficiency exceeding $9 \% . J$. Mater. Chem. A 2016, 4, 877-886.

37. Carey, G. H.; Abdelhady, A. L.; Ning, Z.; Thon, S. M.; Bakr, O. M.; Sargent, E. H. Colloidal Quantum Dot Solar Cells. Chem. Rev. 2015, 115, 12732-12763

38. Ren, Z.; Wang, J.; Pan, Z.; Zhao, K.; Zhang, H.; Li, Y.; Zhao, Y.; Mora-Sero, I.; Bisquert, J.; Zhong, X. Amorphous TiO2 Buffer Layer Boosts Efficiency of Quantum Dot Sensitized Solar Cells to over 9\%. Chem. Mater. 2015, 27, 8398-8405.

39. Sanehira, E. M.; Marshall, A. R.; Christians, J. A.; Harvey, S. P.; Ciesielski, P. N.; Wheeler, L. M.; Schulz, P.; Lin, L. Y.; Beard, M. C.; Luther, J. M. Enhanced mobility $\mathrm{CsPbI}_{3}$ quantum dot arrays for record-efficiency, high-voltage photovoltaic cells. Sci. Adv. 2017, 3, eaao4204.

40. Pan, Z.; Rao, H.; Mora-Seró, I.; Bisquert, J.; Zhong, X. Quantum dot-sensitized solar cells. Chem. Soc. Rev. 2018, 47, 7659-7702.

41. Mora-Seró, I. et. al. Nanoscale Interaction between CdSe or CdTe Nanocrystals and Molecular Dyes Fostering or Hindering Directional Charge Separation. Small 2010, 6, 221-225.

42. Blas-Ferrando, V. M.; Ortiz, J.; González-Pedro, V.; Sánchez, R. S.; Mora-Seró, I.; Fernández-Lázaro, F.; Sastre-Santos, Á. Synergistic Interaction of Dyes and Semiconductor Quantum Dots for Advanced Cascade Cosensitized Solar Cells. Adv. Funct. Mater. 2015, 25, 3220-3226.

43. Hu, L.; Wang, W.; Liu, H.; Peng, J.; Cao, H.; Shao, G.; Xia, Z.; Ma, W.; Tang, J. $\mathrm{PbS}$ colloidal quantum dots as an effective hole transporter for planar heterojunction perovskite solar cells. J. Mater. Chem. A 2015, 3, 515-518.

44. Etgar, L.; Gao, P.; Qin, P.; Graetzel, M.; Nazeeruddin, M. K. A hybrid lead iodide perovskite and lead sulfide QD heterojunction solar cell to obtain a panchromatic response. J. Mater. Chem. A 2014, 2, 11586-11590.

45. Seo, G.; Seo, J.; Ryu, S.; Yin, W.; Ahn, T. K.; Seok, S. I. Enhancing the Performance of Sensitized Solar Cells with PbS/CH3NH3PbI3 Core/Shell Quantum Dots. J. Phys. Chem. Lett. 2014, 5, 2015-2020.

46. Yang, Z.; Janmohamed, A.; Lan, X.; García de Arquer, F. P.; Voznyy, O.; Yassitepe, E.; Kim, G.-H.; Ning, Z.; Gong, X.; Comin, R.; Sargent, E. H. Colloidal Quantum Dot Photovoltaics Enhanced by Perovskite Shelling. Nano Lett. 2015, 15, 75397543.

47. Yang, Z.; Voznyy, O.; Walters, G.; Fan, J. Z.; Liu, M.; Kinge, S.; Hoogland, S.; Sargent, E. H. Quantum Dots in Two-Dimensional Perovskite Matrices for Efficient NearInfrared Light Emission. ACS Photonics 2017, 4, 830-836.

48. $\quad$ Gong, X.; Yang, Z.; Walters, G.; Comin, R.; Ning, Z.; Beauregard, E.; Adinolfi, V.; Voznyy, O.; Sargent, E. H. Highly efficient quantum dot near-infrared light-emitting diodes. Nat. Photonic 2016, 10, 253-257. 
49. Liu, C.; Peng, H.; Wang, K.; Wei, C.; Wang, Z.; Gong, X. PbS quantum dotsinduced trap-assisted charge injection in perovskite photodetectors. Nano Energy 2016, 30, 27-35.

50. Garcia de Arquer, F. P. et.al. Field-emission from quantum-dot-in-perovskite solids. Nat. Commun. 2017, 8, 14757.

51. Moradi, Z.; Fallah, H.; Hajimahmoodzadeh, M. Nanocomposite perovskite based optical sensor with broadband absorption spectrum. Sens. Actuators, A 2018, 280, 47-51.

52. Jung, Y.-K.; Butler, K. T.; Walsh, A. Halide Perovskite Heteroepitaxy: Bond Formation and Carrier Confinement at the PbS-CsPbBr3 Interface. J. Phys. Chem. C 2017, 121, 27351-27356.

53. Yang, Y.; Wang, W. Effects of incorporating $\mathrm{PbS}$ quantum dots in perovskite solar cells based on CH3NH3PbI3. J. Power Sources 2015, 293, 577-584.

54. Dang, V. Q.; Byun, M.; Kang, J.; Kim, C.; Jung, P.-H.; Kim, Y.-D.; Lee, N.-E.; Lee, $\mathrm{H}$. Low temperature fabrication of hybrid solar cells using co-sensitizer of perovskite and lead sulfide nanoparticles. Org. Electron. 2017, 50, 247-254.

55. Sidhik, S.; Esparza, D.; Martínez-Benítez, A.; Lopez-Luke, T.; Carriles, R.; MoraSero, I.; de la Rosa, E. Enhanced Photovoltaic Performance of Mesoscopic Perovskite Solar Cells by Controlling the Interaction between CH J. Phys. Chem. C 2017, 121, 4239-4245.

56. Yang, H.; Zhang, Y.; Hills-Kimball, K.; Zhou, Y.; Chen, O. Building bridges between halide perovskite nanocrystals and thin-film solar cells. Sustainable Energy \& Fuels 2018, 2, 2381-2397.

57. Cha, M.; Da, P.; Wang, J.; Wang, W.; Chen, Z.; Xiu, F.; Zheng, G.; Wang, Z.-S. Enhancing Perovskite Solar Cell Performance by Interface Engineering Using $\mathrm{CH}_{3} \mathrm{NH}_{3} \mathrm{PbBr}_{0.9} \mathrm{I}_{2.1}$ Quantum Dots. J. Am. Chem. Soc. 2016, 138, 8581-8587.

58. Ip, A. H. et. al. Hybrid passivated colloidal quantum dot solids. Nat. Nanotechnol. 2012, 7, 577-582.

59. Dirin, D. N.; Dreyfuss, S.; Bodnarchuk, M. I.; Nedelcu, G.; Papagiorgis, P.; Itskos, G.; Kovalenko, M. V. Lead Halide Perovskites and Other Metal Halide Complexes As Inorganic Capping Ligands for Colloidal Nanocrystals. J. Am. Chem. Soc. 2014, 136, 65506553.

60. Ning, Z.; Dong, H.; Zhang, Q.; Voznyy, O.; Sargent, E. H. Solar Cells Based on Inks of n-Type Colloidal Quantum Dots. ACS Nano 2014, 8, 10321-10327.

61. Lan, X. et. al. 10.6\% Certified Colloidal Quantum Dot Solar Cells via SolventPolarity-Engineered Halide Passivation. Nano Lett. 2016, 16, 4630-4634.

62. Liu, M. et. al. Hybrid organic-inorganic inks flatten the energy landscape in colloidal quantum dot solids. Nat. Mater. 2016, 16, 258.

63. Kulbak, M.; Gupta, S.; Kedem, N.; Levine, I.; Bendikov, T.; Hodes, G.; Cahen, D. Cesium Enhances Long-Term Stability of Lead Bromide Perovskite-Based Solar Cells. $J$. Phys. Chem. Lett. 2016, 7, 167-172.

64. Swarnkar, A.; Marshall, A. R.; Sanehira, E. M.; Chernomordik, B. D.; Moore, D. T.; Christians, J. A.; Chakrabarti, T.; Luther, J. M. Quantum Dot-Induced Phase Stabilization of $\alpha-\mathrm{CsPbI}_{3}$ Perovskite for High-Efficiency Photovoltaics. Science 2016, 354, 92-95.

65. Zhang, X.et. al. Inorganic CsPbI3 Perovskite Coating on PbS Quantum Dot for Highly Efficient and Stable Infrared Light Converting Solar Cells. Adv. Energy Mater. 2018, 8, 1702049.

66. Heo, J. H.; Jang, M. H.; Lee, M. H.; Shin, D. H.; Kim, D. H.; Moon, S. H.; Kim, S. W.; Park, B. J.; Im, S. H. High-Performance Solid-State PbS Quantum Dot-Sensitized 
Solar Cells Prepared by Introduction of Hybrid Perovskite Interlayer. ACS Appl. Mater. Interfaces 2017, 9, 41104-41110.

67. Li, S.-S. et. al. Intermixing-seeded growth for high-performance planar heterojunction perovskite solar cells assisted by precursor-capped nanoparticles. Energy Environ. Sci. 2016, 9, 1282-1289.

68. Han, J.; Luo, S.; Yin, X.; Zhou, Y.; Nan, H.; Li, J.; Li, X.; Oron, D.; Shen, H.; Lin, $\mathrm{H}$. Hybrid PbS Quantum-Dot-in-Perovskite for High-Efficiency Perovskite Solar Cell. Small 2018, 14, 1801016.

69. Galar, P.; Piatkowski, P.; Ngo, T. T.; Gutiérrez, M.; Mora-Seró, I.; Douhal, A. Perovskite-quantum dots interface: Deciphering its ultrafast charge carrier dynamics. Nano Energy 2018, 49, 471-480.

70. Luque, A.; Martí, A. Increasing the Efficiency of Ideal Solar Cells by Photon Induced Transitions at Intermediate Levels. Phys. Rev. Lett. 1997, 78 (26), 5014-5017.

71. Luque, A.; Marti, A.; Stanley, C. Understanding intermediate-band solar cells. Nat. Photonics 2012, 6, 146-152.

72. Blokhin, S. A. et. al. AlGaAs/GaAs photovoltaic cells with an array of InGaAs QDs. Semiconductors 2009, 43, 514-518. 